\title{
AN IMPROVED ACETIC ORCEIN SQUASH METHOD FOR SERIAL CYTOLOGICAL PREPARATIONS
}

\author{
A. E. ZEILINGA \\ Institute of Horticultural Plant Breeding, Wageningen, Netherlands \\ Received 26 April 1956
}

\section{INTRODUCTION}

Since 1951 we have produced tetraploid strains of many kinds of vegetables and fruits by means of colchicine. As an ultimate test for tetraploidy we have always employed the cytological method, and for counting chromosomes it is essential that they are made visible, e.g. by staining.

It is obvious that the number of plants that can be tested in this manner depends on the number of slides prepared and inspected in a given time. Therefore, the method used should meet the following conditions: 1 . It should take little time, hence the procedure should be simple; 2 . It should produce clear pictures of chromosomes in a wide range of plant species; 3 . It should be suitable for continuous serial work.

Of the methods generally employed, e.g. acetic carmine-, acetic lacmoid-, acetic nigrosin- and acetic orcein-squash, the latter as described by LA Cour (1) suited our purpose best. For serial work this method has some shortcomings, but during 1951 we succeeded in eliminating them. These shortcomings include:

a. Maceration and rinsing in water as used in combination with hydroxyquinolin (see Prakken \& Swaminathan, 2) takes too long.

$b$. When transferring material from staining bath to slide, the meristematic tissues, especially from tender root tips, are readily lost, leaving only coarse material without dividing cells.

c. The stain penetrates too slowly, resulting in partly coloured preparations, if applied after maceration and rinsing.

d. It is very difficult to regulate the temperature of the staining bath by staining in a watch glass; this results in many understained or, even worse, overstained preparations.

$e$. If after squashing in acetic orcein the material remains in this medium, the staining will be satisfactory for only one or two days. As the acetic acid gradually evaporates from the solution the orcein precipitates in the plasm, thus spoiling the entire preparation.

$f$. The red colour of the orcein solution is in poor contrast with the orange-brown coloured nuclei. Working with such preparations is trying to the eyes.

The improved technique described below enables us to make the preparations quicker and better than before. Thirty are easily made within one and a half hours. According to our technique, we stain on the slide in a drop of $2 \%$ orcein solution in $45 \%$ acetic acid adding $\mathrm{N} \mathrm{HCl} 1$ to 9 . Instead of squashing in acetic orcein we use Fast Green FCF solution, $0.1 \%$ in $45 \%$ acetic acid. It produces a fine counterstain, as it colours the plasm with a light bluish tinge. 


\section{Procedure}

Our technique is as follows:

1. Fix in alcohol-acetic acid 3:1, preferably for 2 to 3 hours.

2. Put fixed material on a clean slide; remove all superfluous parts.

3. Add a drop of orcein stain solution after fixing fluid has evaporated.

4. Allow stain solution to penetrate the entire material for one minute and afterwards warm gently over a spirit flame. Avoid boiling. Heating once is generally sufficient.

5. Leave the slide for 10 minutes. The solution now slowly dries. During this process the staining gradually intensifies and maceration goes on.

6. Before it is quite dry the material should be pushed to a clean place on the slide with a piece of filter paper, and the staining solution should be blotted off.

7. Put some drops of Fast Green F.C.F. on the material and leave it in the counterstaining solution for a minute or longer.

8. Put the cover slip on and squash the material under it. A match stick is very convenient for doing this. After tapping gently, the cells of the material flow easily to all sides, forming a closed layer one cell thick.

9. Blot off the superfluous solution using several sheets of blotting paper. Care should be taken not to move the cover slip. Press firmly after slight heating if flattening of the cells is desired.

10. Seal with paraffin wax.

\section{SERIAL WORK}

To prepare a series of preparations we arrange 30 slides on the table and each is given the proper serial number. On one end of the slide we then put the fixed material e.g. a root tip, part of a young leaf or an anther. If 10 slides are provided with material, we put a drop of stain on the first series of five. Then the third series of five slides is provided with material and then the second series with stain. After 20 slides have thus been treated one can start heating the slides. So we go on till all the 30 slides have had their turn.

The first preparations are sufficiently stained now, so we start pushing the material to the centre of the slide, blotting off the superfluous orcein solution and adding Fast Green, after 5 slides have been handled. Squashing can now be started. Care should be taken not to lose too much fluid from under the cover slip, while the material has not been entirely squashed. As long as the fluid film under the cover slip is thick enough the slip can be moved without damaging the cells. Sometimes this moving is necessary in order to separate the individual cells.

The slides may be left for some time after squashing. When 10 are ready, blot off and seal instantly with paraffin wax.

Our sealing tool is a copper wire about $3 / 16$ inches thick, fastened to a wooden handle. The end of the wire is bent at a right angle over a length of half an inch and flattened, making a sharp edge, thus simulating a small soldering iron. 


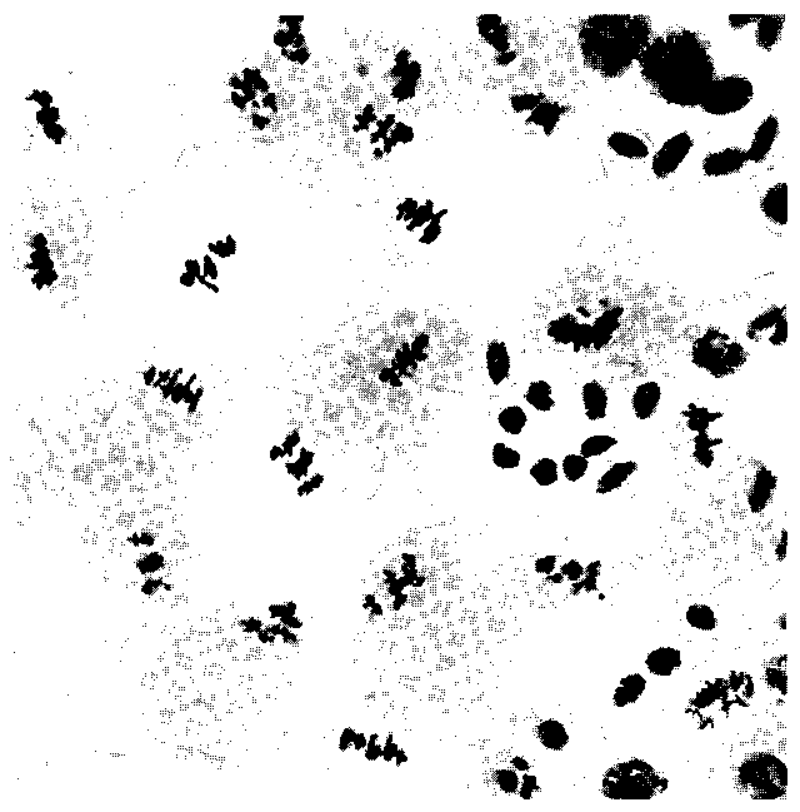

Fig. 1. Anemone coronaria, Mi. Acetic-orcein squash in Fast Green. Photograph TAKEN ONE MONTH AFTER PREPARING THE SLIDE $480 \times$

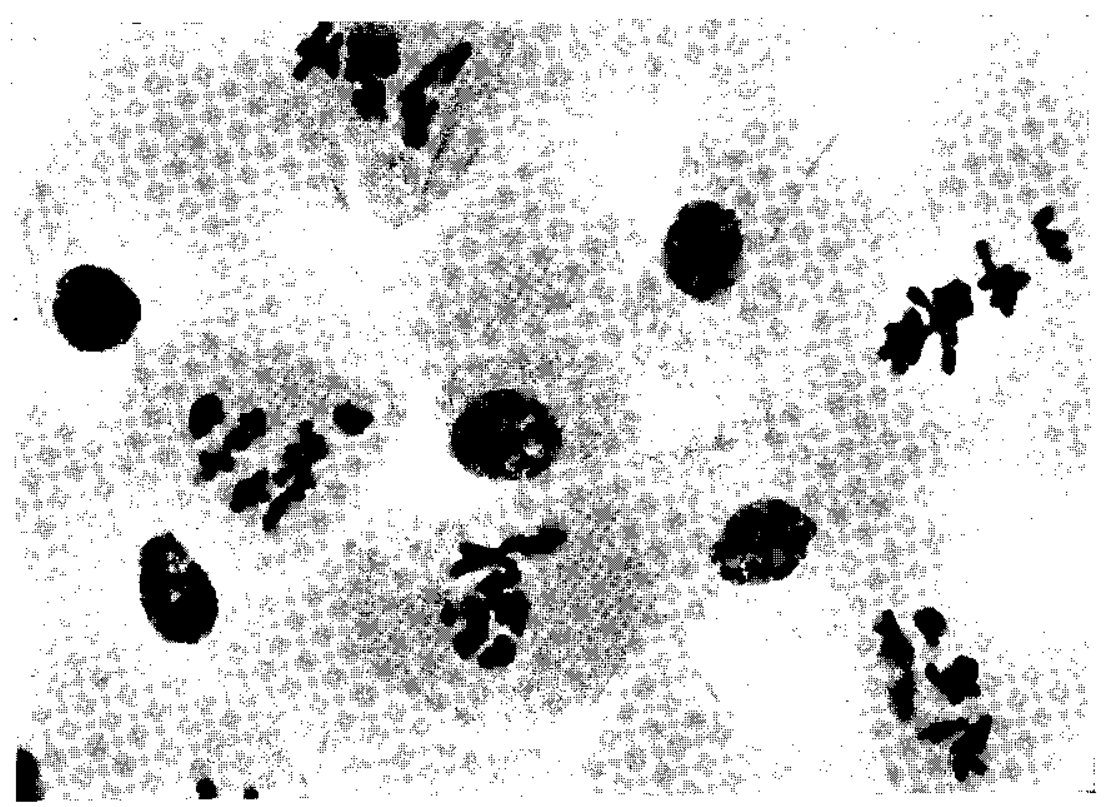

Fig. 2. Anemone coronaria, Mi. Acetic-Orcein squash in Fast Green. Photograph TAKEN ONE MONTH AFTER PREPARING THE SLIDE $1200 \times$ 
A. E. ZEILINGA

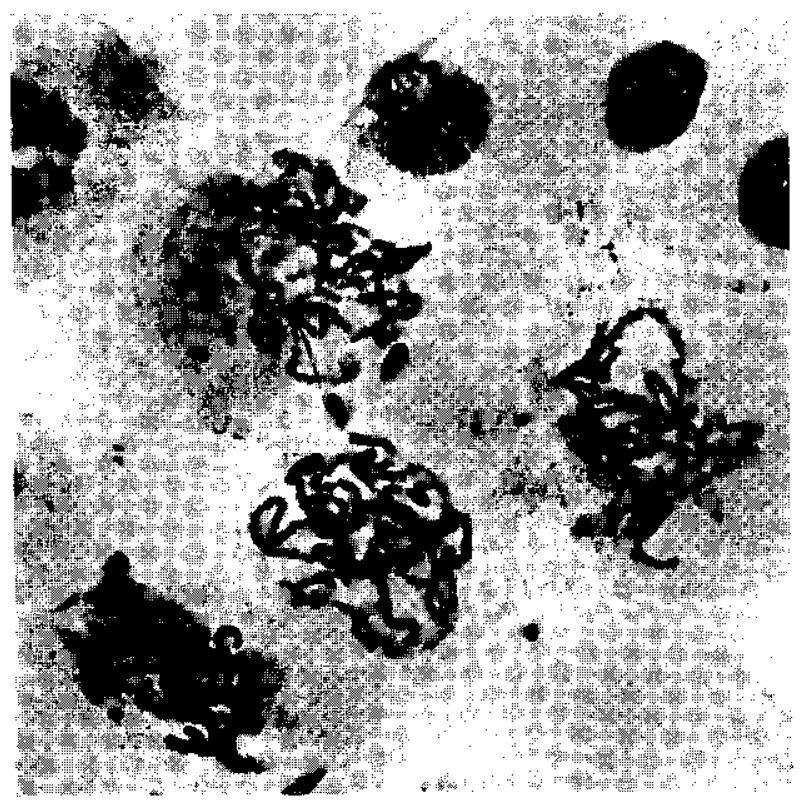

Fig. 3. Anemone coronaria, Pachytene. Acetic-orcein sQuash in fast Green. PHOTOGRAPH TAKEN ONE MONTH AFTER PREPARING THE SLIDE $1200 \times$

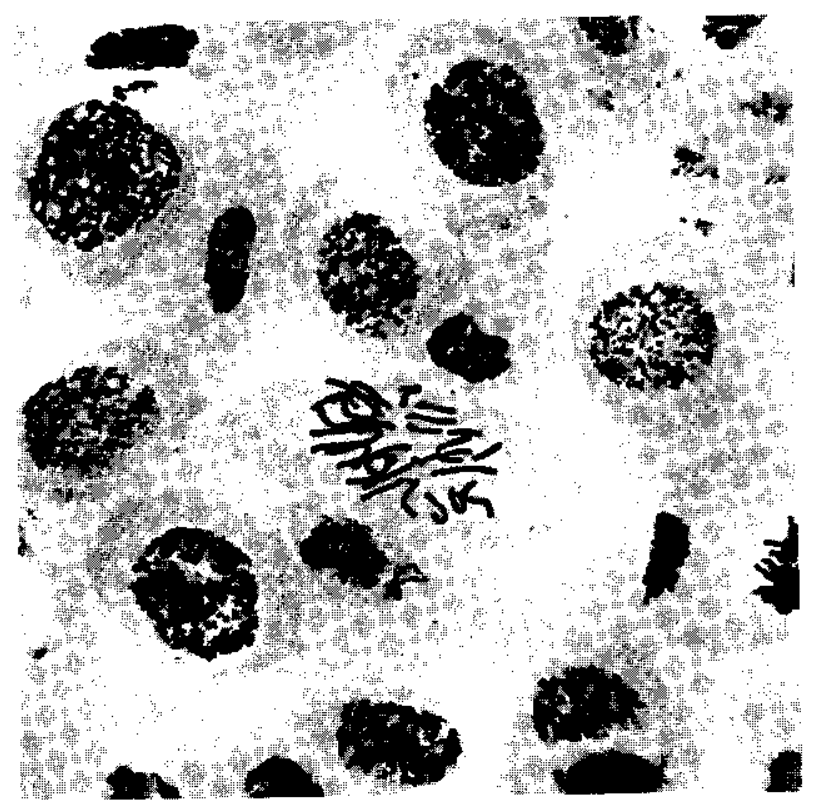

Fig. 4. Lilium $\times$ “Orange Triumph". RT. ACETIC-OrCeIN SQUaSH IN FAST Green. Photograph of FReshly PREPARED SLIDE $480 \times$ 
FIG. 5.

Lilium $\times$ "Orange Triumph". RT. ACETIC-ORCEIN SQUASH IN FAST Green. Photograph of a freshly PREPARED SLIDE $1200 \times$.

\section{CONCLUDING REMARKS}

We have obtained excellent results with a great variety of plants by means of the technique described above. In some species it is advisable to stain twice. So after stage 7 of the technique one starts again at 5 . Never heat a drop of acetic orcein twice, it will get too hot. After heating a fresh drop of acetic orcein on a slide it will contract like a drop of water on a greasy surface. This contraction is an indication that the drop has attained the right temperature. When heating a drop a second time, it does not contract so easily and becomes too hot before showing contraction. Such overheated slides are worthless. The chromosomes in such preparations have lost their proper shape, in the worst case only some drops of chromatin instead of chromosomes can be seen.

The nuclei and chromosomes in our preparations are coloured deep orange-brown; the plasm, being bluish green or bluish grey, affords a sharp contrast with the chromosomes. Faultlessly prepared slides keep for several weeks. They, can be made permanent in the usual way.

To give an impression of the results that can be obtained with our technique we present some microphotographs. Figs. 1 to 3 show slides one month after being prepared. Figs. 4 to 5 depict fresh preparations. Slides kept longer than a month become gradually opaque.

It is regretted that the colour contrast is not shown in the photographs.

\section{SUMmaRY}

As a final test for colchicine-induced tetraploid plants the cytological method is most reliable. However, cytological testing can only be done in a satisfactory manner if a large series of preparations can be prepared in a relatively short time. 


\section{A. E. ZEILINGA}

It is shown how, after some simplifications and improvements with LA CouR's acetic orcein staining, a trained person can in about one and a half hours prepare a series of 30 preparations which keep for one month or longer.

Staining and maceration are done on the slide; the material is squashed and mounted in acetic Fast Green. Fast Green enhances the contrast and the preparations mounted in this solution do not deteriorate so quickly as when mounted in orcein solution.

\section{SAMENVATTING}

Een verbeterde orceine-squash methode voor het vervaardigen van series cytologische preparaten

Voor het maken van series cytologische preparaten, zoals door ons worden gebruikt voor het testen van met colchicine behandelde planten, is een snelle en betrouwbare cytologische techniek zeer belangrijk.

Het voorgaande is een uiteenzetting, hoe, na enkele vereenvoudigingen en verbeteringen, met de orceine kleuring van LA CoUR, een geoefend persoon, zonder inspanning, een serie van 30 preparaten, die \pm een maand goed bruikbaar blijven, in $1 \frac{1}{2}$ uur vervaardigt.

Het essentiële van de methode is, dat de kleuring en de maceratie op het dekglas uitgevoerd worden en dat in een Fast Green oplossing wordt ingesloten, hetgeen de houdbaarheid bevordert. Fast Green verhoogt tevens het contrast in het preparaat.

\section{REFERENCES}

1. LA Cour, L., Acetic-orcein: a new strain-fixative for chromosomes. Stain Technology 16 (1941): $169-174$.

2. Prakken, R. and Swaminathan, M. S., Experience with the hydroxyquinoline smear method. Med. Landbouwhogeschool 50 (1951): 137-140. 


\section{MEDEDELINGEN ${ }^{1}$}

\section{VAN HET INSTITUUT VOOR DE VEREDELING VAN TUINBOUWGEWASSEN}

38. Banga, O. en M. Keuls. Practijkproeven zomerwortelen 1949-1950. Juli 1952 Uitverkocht

39. Kronenberg, H. G. Veredelingswerk met de aardbei op het I.V.T. Octaber 1952 ....... Uitverkocht

40. Floor, J. Proeven met vermeerdering door entstekken, October 1952

41. Banga, $O$. Some factors in the growth rate of red garden beets. November 1952

42. Sneep, J. Practijkproeven met Westlandse Boerenkool 1949-1950 en 1950-1951. December 1952 f

43. Een bos enthoutjes. Januari 1953 . Ranga, $O$. Practijkproeven met Ronde Rode Radijs 1951-1952. Februari 1953....... . f 0,65

45. Gerritsen, C. J. De rassenkeuze bij de Walnoot. Maart 1953

46. Kronenberg, H. G. De veredeling van KleinFruit in de Ver. Staten van Amerika ........

47. Banga, O. en M. Keuls. Practijkproeven met Berlikumer Wortel 1940. April 1953.

48. Gerritsen, C. J. Welke kersen moiten we planten. April 1953

49. Banga, O., M. Keuls en M. Wattel. Practijkproeven met Flakkeese Winterwortel 1950-1951. Mei 1953

50. Algemene Veredelingsdagen '1952. Verslag van voordrachten en discussies. Juni 1953 .

51. Sneep, J. Practijkproeven met Spitskool 19491950 en 1950-1951. Juli 1953 . . . . . - .

2. Boom, B. K. International reglement voor de naamgeving van gekweekte planten . . . . $\dot{\text { Kp }} \cdot$

53. Kronenberg, H. G. en F. Garretsen. Opbrengstproeven met aardbeiklonen. November 1953

54. Veredelingsdag Groentegewassen 1953. Versiag van voord rachten en discussies. December 1953.

55. Floor, J. Planten in plastic. Januari 1954 . Vitverkocht

56. Banga, $O$. Taproot-problems in che breeding of root vegetables.

57. Jensma, J. R. en A. Kraai. Practijkproeven met Rode Kool 1950-1951. Juni 1954 ......... f1,10

58. Jensma, J. R. en A. Kraal. Practijkproeven met Spruitkool 1950-1951. Juli 1954........ . f 0,85

59. Veredelingsdag Fruitgewassen $1954^{\circ}$. Verslag van voordrachten en discussies. Augustus 1954 ... . . f 0,95

60. Kraai, A. The use of Honey-bees and Bumble-bees in breeding work. September $1954 \ldots \ldots$ f 0.45

61. Jensma, J. R. en A. Kraai. Practijkproeven met Witte Kool 1952-1953. Februari 1955 ....... f 1,35

62. Banga, O. en J. W. de Bruyn. Selection of Carrots for Carotene Content. Februari 1955 . . . . . . . . f 0.25

63. Kronenberg, H. G. en L. M. Wassenaar. Practijkproeven met aardbeirassen 1952-1954. April 1955 * f 0.90

64. Keuls, M. and J. W. Sieben. Two statistical problems in plant selection. April 1955 . . . . . . . f 0,35
65. Banga, 0 . The Institute of Horticultural Plant Breeding. April 1955 ........... f 0,25 66. Banga, $\mathbf{O}$. Uienveredeling met gebruikmaking van inteelt en herstel door heterosis. Juni 1955 ..... f 0,30

67. Banga, O. Carrot yield analysis. September 1955 . 0,30

68. Banga, O., J.W. de Bruyn and L. Smeete. Selection of carrocs for carotene content. Il Sub-normal content at low temperature. September 1955. . . . . . . . f 0,25

69. Braak, $\}$. P. Effect of temperature and light on June Yellows in strawberries. September $1955 \ldots$. . . 0.25

70. Banga, 0 . De ontwikkeling van de rassensituatie bij groentegewassen. Oktober $1955 \ldots$...... f 0,25

71. Bruyne, A.S. de. Tendenzen in de ontwikkeling van het Nederlandse fruitsortiment. Oktober 1955 . . . f 0.40

72. Banga, O. Praktijkproeven met Knolselderij 1953-1954. November 1955 . . . . . . . . . . . . f 0,30

73. Floor, J., Proeven met stekken onder waterverneveling. April 1956 ; . . . f 1, -

74. Andeweg, J. M. en J. H. Ruyten. Praktijkproeven met Tomaten 1954-1955. April 1956 ..... . . f 0,40

75. Andeweg, J. M. en A, van Steenbergen. Praktijkproeven met Stoksnifbonen 1953-1954. Mei 1956 . . . f 0,35

76. Banga, O. en J. L. van Bennekom. Praktijkproeven met Ronde Witpunt Radijs 1953-1954. Mei 1956. . f 0.55

77. Smeets, L. and Hester G. Kronenberg. Runner formation on strawberry plants in autumn and winter. Smeets, L. Runner formation on strawberry plants in autumn and winter. II. Influence of the light intensity on the photoperiodical behaviour. Juni 1956 ... f 0,30

78. Smeets, $L$. Influence of the temperature on runner production in five strawberry varieties. Juni 1956 . . f 0,25

79. Smeets, L. and L. M. Wassenaar. Problems of heat spot in Fragaria vesca $L$. when indexing strawberry selections for viruses. Juni 1956

80. Banga, $O$, and $W$ de Bruyn, Selection of carro carotene content. III Planting distances and ripening librium of the roots. Juni $1956 \ldots . . .20,35$ 1. Banga, O. International conference on the improvement and on the standardization of vegetable varieties at Wageningen, Netherlands, on August 26 and 27, 1955. August 1956 . 075

82. Floor, J. Proeven met varmeerdering van houtige gewassen. September 1956 . 0.80

83. Gerritsen, C.J. Improvement of the cherry varieties used in the Netherlands. Oktober 1956 . . . . . f 0,35

84. Gerritsen, C. J. Research offered new possibilities for nut growing in the Netherlands. Oktober 1956... f 0,25

85. Andeweg, J. M. The breeding of scab-resistant frame cucumbers in the Netherlands. Oktober 1956 . . . 0,30

86. Zeilinga, A. E. An improved acetic orcein squash method for serial cytological preparations. Oktober 1956 . f 0,20

\section{PERSBERICHTEN UITSLAGEN PRACTIKRROEVEN}

10- 3-'50. Uitslag Practijkproeven Wortel Berlikumer 1949.

29-11-'50. Uitslag Practijkproeven Bak- en Zomerwortelen 1949-1950.

29-11-'50. Uitslag Practilkproeven Platronde en Ronde Kroten 1949-1950.

22-12-'50. Uitslag Practijkproeven Pronkbonen 1950.

11- 3-'51. Uitslag Practijkproeven Westlandse Boerenkool 1949-1950.

3- 9-'51. Uitslag Practijkproeven Spitskool 1950-1951.

7-12-'51. Uitslag Practijkproeven Flakkeese Winterwortel 1950-1951.

23- 1-'52. Uitslag Practijkproeven Vroege en Herfsc Rodekool 1950-1951.

31- 3-'52. Uitslag Practijkproeven Spruitkool 1950-1951.

4-11-'52. Uitslag Practijkproeven Ronde Rode Radijs 1951-1952.

4-11-'52. Uicslag Practijkproeven Vroege Rijspeulen 1951-1952.

25-11-'52. Uitslag Practijkproeven Lange Kroten 1951-1952.

23- 1-'53. Uitslag Practijkproeven Radijs Rond scharlakeni ode Extra Kortloof 1951-1952.

13- 5-'53. Uitslag Practijkproeven Bewaar Rode Kool 1951-1952.

10- 9-'53. Uitslag Practijkproeven Vroege Witte Kool 1952-1953.

18-12-'53. Uitslag Practijkproeven Herfst Witte Kool 1952-1953.

3- 6-'54. Uitslag Practijkproeven Bewaar Witte Kool 1952-1953.

17-11-54. Uitslag Practijkproeven Stoksnijbonen 1953-1954

2-12-54. Uitslag Practijkproeven Ronde Rode Witpunt Radijs 1953-1954.

12- 2-'55. Uitslag Practijkproeven Knolselderij 1953-1954.

1. 9-'55. Uitslag Practijkproeven Vroege Groene Savoye Kool 1954-1955.

14-11-'55. Uitslas Practijkproeven Tomaten 1954-1955.

27 12-'55. Uitslag Practijkproeven Witlof vroege trek 1954-1955.

2-3-56. Uitslag Practijkproeven Witlof middelvroege crek 1954-1955.

5-3-'56. Uitslag Practijkproeven Schorseneren 1954-1955.

28- 5-'56. Uitslas Practijkproeven Savoye Kool 1954-1955.

28- 5-'56. Uitslag Practijkproeven Wielof koude kuil en meilor 1954-1955.

30- 7.'56. Uitslag Practijkproeven Tuinbonen 1955-1956.

1. 9-'56. Uitslag Praktijkproeven Amsterdamse Bakwortel 1955-1956

1- 9-'56. Uitslag Praktijkproeven Vroege Rode Kool 1956

1- 9-'56. Uitslag Praktijkproeven Platronde Kroten 1955-1956

Zijn geplaatst in diverse cuinbouwbladen. 


\section{RASSENLIJSTEN ${ }^{1}$ ) \\ UITGEGEVEN DOOR HET INSTITUUT VOOR DE VEREDELING VAN TUINBOUWGEWASSEN}

Achtste Beschrijvende Rassenlifst voor Fruit. 1957. . . f 1,75 $\begin{array}{r}\text { Achtste Beschrijvende Rassenlijst voor Groentegewas- } \\ \text { sen. 1956. Redacteur Dr. O. Banga . . . . . . } 1,75\end{array}$

JAARVERSLAGEN ${ }^{1}$ )

VAN HET INSTITUUT VOOR DE VEREDELING VAN TUINBOUWGEWASSEN

Jaarverslag $1950.1(1951) \ldots \ldots \ldots \ldots \ldots \ldots \ldots \ldots$ Uitverkoche

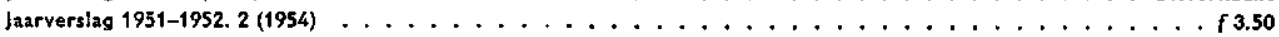

\section{PUBLIKATIES VAN HET INSTITUUT VOOR DE VEREDELING VAN TUINBOUWGEWASSEN IN ANDERE ORGANEN OF IN BOEKVORM EVENTUEEL IN SAMENWERKING MET ANDERE INSTELLINGEN 2)}

De publikaties, waarvan prijs èn uitgever worden vermeld zijn verkrijgbaar in de boekhandel. Overigens wende men zich tot de opgegeven bronnen of cot de bibliotheek van het I.V.T. EJzenga, $\mathbf{G}$. De selectie van Angelica-wortel op wortelgewicht V.N.K.-Nieuws 1955: 34-39, 42-47.

Gerritsen, C. J. De selectie van (okker)-noten. Dendrologisch laarboek 1954 : 40-43.

Jensma, J. R. Mei in Mechelen. Groenten en Fruit 10, 1955 1292.

Floor, J. en P. A. Wezelenburz. Planten in plastic. Uitg. I.V.T. Juni 1955,10 p. 10,25

Banga, 0 . De plantenveredeling als factor in de strijd om het bestaan. Extra nummer Landbouwkundig Tijdschrift Me 1955 en Zaadbelangen 9, 1955: 173-174, 187-188.

Kronenberg. H. G. Ervaringen met aardbeirassen in 1955 Groenten en Fruit 11, 1955: 138.

Elzenga, $\boldsymbol{G}$. Het kweken van plantmateriaal van Valeriaan. V.N.K.-Nieuws 1955: 70-72.

Jensma, J.R. is spits + vroeg + groen $=$ Vroege Groene Spitse? Groenten en Fruit 11, 1955: 221.

Elzenga, G. Digicalis lanata. V.N.K.-Nieuws 1955: 87-91.

Boom, B. K. Nores on culcivated plants. Acca Bocanica Neerlandica 4, 1955: 167-171.

Banga, O. Het XIVe Internationale Tuinbouwcongres. Zaadbelangen 9, 1955: 232-233.

Gaag, H. C. v. d. Het kweken en het gebruik van vleesvliegen bij het veredelingswerk. Zaadbelangen 9, 1955: 233-236.

Elzenga, $\mathbf{G}$. Veredelingsproblemen bij Angelica archangelica. Meded. Dir. Tuinbouw 18, 1955: 748-752.

Floor, J. Problemen bij de selectie van rozenonderstammen. Meded. Dir. Tuinbouw 18, 1955: 706-710.

Kronenberg, H. G. (I.V.T.) en J. J. Doesburg (I.B.V.T.). Meer aandacht voor het vitamine-C-gehalte van $z$ warte bessenrassen. Groenten en Fruit 11, 1955: 365. De Fruitteelt 45, 1955: 869 .

Banga, O. De benaming van rassen. Zaadbelangen 9, 1955 : 247-248.

Boom, B. K. Cotoneaster wardii en verwante soorten. De Boomkwekerij 11, 1955: 3.

Gerritsen, C. J. Zit er wat in de teelt van hazelnoten? De Fruitteelt 45, 1955: 865 .

Kronenberg, H, G. Aardbeien. Wat moeten we toch planten? De Fruitteelt 45, 1955 : 866-867.

Gerritsen, C. J. Gaat $U$ kersen planten? De Fruitteelt 45 1955: $909-910$.

Burg, J. P. L. L. A. en G. Elzenga. Rapporc over een studiereis aangaande de teelt en verwerking van geneeskrachtige en aromatische gewassen in Duirsiand en Frankrijk $116 \mathrm{c} / \mathrm{m}$ 31 augustus 1955). V.N.K.-Nieuws, september 1955: 92-99.

Boom, B. K. Sorbus pratci en S. koeneana. De Boomkwekerij 11, 1955: 27.

Elzenga G. Het rooien van de wortels van Angelica en $V$ aleriaan. V.N.K.-Nieuws, november 1955: 110-111

Elzonga, G. Pepermunt opnieuw inplanten. V.N.K.-Nieuws, noyember 1955: 112

Boom, B. K. Vraagstukken rondom het Cotoneaster-sortimen. De Boomkwekerij 11, 1955, 41-42.

Jensma, J. R. Rassenkeuze bij bloemkool. Groenten en Fruit 11, 1956: 721 .

Bruyne, A. S. de. Nieuwe appelrassen tor James Grieve. De Fruitwereld 1, 1956; no 4; 8-9.

Boom, B. K. Cercidiphyllum. De Boomkwekerij 11, 1955: 27.

Broertjes, C. Reactie op vraagstukken rondom het Cotoneastersortiment. De Boomkwekerij 11, 1956: 67-68. roerties, C. Veredeling op ziekteresistentie bij rozen. De Boomkwekerij 11, 1956: 73.

Boom, B. K. Acer platanoides, reicenbach' en ,rubrum' De Boomkwekerij 11, 1956: 74.

Bruyn, J.W. de. De exportcontrole van kruiden in 1955. V.N.K,-Nieuws, januari 1956: 134-135.

Boom, B. K. Buxus, buksus of buks. De Boomkwekerij 11, 1956: 80-81.

Boom, B. K. Drie nieuwe wilgen. De Boomkwekerij 11, 1956: 81-82.

Boom, B. K. Enkele bontbladige bomen. De Boomkwekerij 11, 1956: 88 .

Boom, B. K. Een nieuwe monographie over het geslacht Philadelphus. De Boomkwekerij 11, 1956: 96-97.

Gerritsen, C. J. Zal de noot een deugd worden? De Fruitwereld 1, 1956; no. 14: 5.

Banga, O. Enkele opmerkingen naar aanleiding van een internationale conferentie. Zaadbelangen 10, 1956: 101-102.

Kronenberg, H. G. Strawberry growing in the Netherlands. American Fruit Grower 76, 1956; no. 4: 77.

Elzenga, G. Lobelia inflata. V.N.K.-Nieuws, maart 1956: 163 166.

Boom, B. K. Variëteit en cultivar. De Boomkwekerij 11 , 1956: 112-113.

Andeweg, ). M. Vroegrijpende moneymaker's. Zaadbelangen 10, 1956: 145.

Boom, B. K. Verwarring over de plantennamen. Vakblad voor de Bloemisterij 11, 1956: 130-131.

Gijsbers, J. W. Ruimtebesparing bij de opberging van dia's en negatieven. Meded. Dir. Tuinbouw 19, 1956: 298-300

en g. K. Over een verzameling prijscouranten. De Boomkwekerij 11, 1956: 128-129.

Boom, B. K. Een Amerikaan over Boskoop. De Boomkwekerij 11, 1956: 130.

Huyskes, J. A. Klauwenselectie bij asperges geeft goede resultaten. Boer en Tuinder (Land en Vee) 10, 1956; no. $482: 17$.

Koot, Y. v. en J. M. Andewer. De groenteteelt in Amerika. 's-Gravenhage, C.O.P., 1956. 149 blz. $17,00$.

Banga, $O$. Kweker en overheid in de sector groentezaden. Zaadbelangen 10, 1956: 189-190.

Kho, $\mathbf{Y}, \mathbf{O}$. Opbrengstvermindering en kiemkrachtverlaging van wortelzaad als gevolg van aantasting door wantsen. Zaadbelangen 10, 1956: 193-194.

Elrenga, G. Digicalis lanata Ehr. V.N.K.-Nieuws 1956: 167-170, 193-199.

Andeweg, J. M. Rationalisatie en rassenkeuze. Groenten en Fruic 12, 1956 : 111.

Kho, Y. O. en J. P. Braak. Opbrengstvermindering en kiemkrachtverlaging van wortelzaad als gevolg van aantasting door wantsen. Meded. Dir. Tuinb. 19, 1956: 440-445.

Kronenberg, H. G. Praktijkproeven met aardbeien in 1956. De Tuinderij 36, 1956, no 33: 1-3. Groenten en Fruit 12, 1956: 177.

Floor, J. en P. A. Wezelenburg. Stekken onder plastic. De Boomkwekerij, 11, 1956: 174-175.

Terpstra, W. Some factors influencing the abscission of debladed leaf petioles. Acta Botanica Neerlandica 5, 1956: 157-170

Bruyne, A. S. de. Trends and developments in Dutch varieties. The Commercial Grower 1956, no 3165: 419-422.

1) Zolang de voorraad strekt kunnen deze publikaties franco worden toegezonden, na ontvangst van het vermelde bedrag op giro no. 425340 van het Instituut yoor de Veredeling van Tuinbouwgewassen, S. L. Mansholtlaan 15 te Wageningen onder vermelding van wat verlangd wordt; ook bestaat de mogelijkheid deze publikaties uit de bibliotheek van het I.V.T. te lenen.

2) Eerder verschenen publikaties zijn vermeld achterin in de Mededelingen nos $1 \mathrm{t} / \mathrm{m} 70$. 